\title{
On the existence of another source of heat production for the earth and planets, and its connection with gravitomagnetism
}

\author{
Alexandre Chaloum Elbeze
}

\begin{abstract}
Recent revised estimates of the Earth's surface heat flux are in the order of 47 TW. Given that its internal radiogenic (mantle and crust) heat production is estimated to be around 20 TW, the Earth has a thermal deficit of around 27 TW. This article will try to show that the action of the gravitational field of the Sun on the rotating masses of the Earth is probably the source of another heat production in order of 54TW, which would satisfy the thermal balance of our celestial body and probably explain the reduced heat flow Qo. We reach this conclusion within the framework of gravitation implied by Einstein's special and general relativity theory (SR, GR). Our results show that it might possible, in principle, to calculate the heat generated by the action of the gravitational field of celestial bodies on the Earth and planets of the Solar System (a phenomenon that is different to that of the gravitational tidal effect from the Sun and the Moon). This result should help physicists to improve and develop new models of the Earth's heat balance, and suggests that contrary to cooling, the Earth is in a phase of thermal balance, or even reheating.
\end{abstract}

Keywords: Earth's surface heat; New source of heat; Reduced heat flow; Earth's thermal balance; Gravitomagnetism; Radioactive heating; Bulk Silicate Earth (BSE)

\section{Introduction}

Approximately fifty per cent of the heat generated by the Earth is thought to be produced by the radioactive decay of elements such as uranium, thorium and their isotopes. Geophysicists estimate heat flow from the Earth's interior to be in the order of 47 TW (Davies and Davies 2010), which is similar to, but slightly higher than previous estimates (e.g. Pollack et al. $1993-44.2 \mathrm{TW} \pm 1 \mathrm{TW}$ and Jaupart et al. $2007-46$ TW \pm 3 TW ).

What still remains to be understood is the quantity of heat generated from the Earth's primitive heat and the heat produced through the decay of radioactive elements found in the mantle. The most popular model of radioactive heating is based on the Bulk Silicate Earth (BSE) model (McDonough and Sun 1995), which assumes that radioactive materials, such as uranium and thorium, are found in the Earth's lithosphere and mantle but not in its iron core. The BSE model also states that the amount of

Correspondence: A77190@bezeqint.net

University Pierre et Marie Curie, Campus Jussieu, Pyramide Patio-Students, 24-14-13-23, 4, place Jussieu, 75252 Paris cedex 05, France radioactive material can be estimated by studying igneous rocks formed on the Earth and the composition of meteorites.

From this model scientists believe that approximately 20 TW (Mareschal JC et al. (1999)) of heat is created by radioactive decay (Palme and O'Neil 2003 and Bellini et al. 2010), comprised of around $8 \mathrm{TW}$ from uranium $\left({ }^{238} \mathrm{U}\right), 8 \mathrm{TW}$ from thorium $\left({ }^{232} \mathrm{Th}\right)$ and $4 \mathrm{TW}$ from potassium $\left({ }^{40} \mathrm{~K}\right)$. Of this, $7 \mathrm{TW}$ is believed to be created in the Earth's crust and 13 TW in the mantle.

At the same time around $8 \mathrm{TW}$ has been attributed to core dissipation in solid earth. Other heat sources have also been suggested; $39 \mathrm{TW}$ of surface heat flux has been attributed to mantle convection processes, which include approximately $1 \mathrm{TW}$ of latent crystallization heat at the inner core boundary (gravitational energy released by the compression of the core would be of the same order), and residual heat from planetary accretion. Although this initial heat may have rapidly dissipated through the Earth's superficial layers, slower internal processes would still 
continue even today (according to some authors this energy has already dissipated).

The heat dissipated by the Earth's mantle is believed to be around $39 \mathrm{TW}$, while internal heat production is thought to be up to $21 \mathrm{TW}$. In other words, if the Earth dissipates more heat than it produces, it is cooling. The difference of $18 \mathrm{TW}$ (Table 1, Jaupart et al. 2007) can be explained by the secular cooling of the mantle.

In this paper we argue that there is another potential source of heat that should be taken into account. This heat is created in the Earth's various layers, mainly the inner and outer core and the mantle (Figure 1) and is the result of the gravitational action of stars (in particular the Sun) and planets on the Earth (and should not be confused with tidal action). We argue that the gravitational influence of the Sun is primordial and far more significant than that of the other planets in the Solar System.

Our argument is based on earlier work (Elbeze 2012) and takes as a starting point results related to the gravitomagnetism framework implied by Einstein's general relativity theory (GR).

\section{The lense-thirring effect}

According to Einstein's GR theory (Einstein 1950), the action of the gravitational potential $\mathrm{U}$ of a given distribution of mass-energy is described by the coefficients $\mathrm{g}_{\mu, v}$, $\mu, \nu .=0,{ }_{-} \_, 2, \_3$, of the space-time metric tensor. They are determined by solving the fully non-linear field equations of Einstein's GR theory for the mass-energy content. These equations can be made linear in the weak-field $\left(U / c^{2}<<1\right.$,

Table 1 Mantle energy budget: preferred value and range

\begin{tabular}{lcc}
\hline & TW & TW \\
\hline Oceanic heat loss $\left(300 \times 10^{6} \mathrm{~km}^{2}\right)$ & 32 & $30-34$ \\
Continental heat loss $\left(210 \times 10^{6} \mathrm{~km}^{2}\right)$ & 14 & $13-15$ \\
Total surface heat loss $\left(510 \times 10^{6} \mathrm{~km}^{2}\right)$ & 46 & $43-49$ \\
Radioactive sources (mantle + crust) & 20 & $17-23$ \\
Continental heat production (crust + lith. mantle) & 7 & $6-8$ \\
Heat flux from convecting mantle & 39 & $35-43$ \\
Radioactive heat sources (convecting mantle) & 13 & $9-17$ \\
Heat from core & 8 & $5-10^{a}$ \\
Tidal dissipation in solid earth & 0.1 & \\
Gravitational energy (differentiation of crust) & 0.3 & \\
Total input & 21 & $14-27$ \\
Net loss (mantle cooling) & 18 & $8-29$ \\
Present cooling rate, $\mathrm{K} \mathrm{Gy}^{-1}$ & 118 & $53-190$ \\
Present Urey ratio & \\
\hline
\end{tabular}

${ }^{a}$ This range includes estimates from core thermodynamics and inference from the perovskite-post-perovskite phase diagram.

${ }^{b}$ Urey ratio for the convecting mantle, leaving out crustal heat sources from both heat loss and heat production. The distribution in the range is barely known for most cases and the preferred value is simply the middle one. The cooling rate is computed assuming $C p=1200 \mathrm{JK}^{-1} \mathrm{~kg}^{-1}$. where $c$ is the speed of light in vacuum) and slow-motion $(v / c<<1)$ approximation (see ML Ruggiero and Tartaglia 2002), valid for the Solar System, and appear similar to the linear Maxwell electromagnetism equations and the noncentral force $F_{L T}$, defined as follows:

$$
F_{L T}=-2 \cdot m \cdot\left(\frac{\vec{v}}{c}\right) \times B_{g}
$$

Eq. (1) shows the force acting on a test particle of mass $m$ induced by the post-Newtonian component $\boldsymbol{B}_{g}$ (De Sitter W (1916a)) of the gravitational field in which the particle moves with velocity $v . \boldsymbol{B}_{g}$ is determined by the mass currents of the matter-energy distribution of the source with mass $M$ and comes from the off-diagonal components $g_{o i} i=1,2,3$ of the metric tensor. The gravitational effects induced by mass displacements are collectively named gravitomagnetism. For a central rotating body of mass $M$ with angular momentum $S$ (parallel to the $\vec{z}$ axis) radius $r$, and the Newtonian gravitational constant $G$ the gravitomagnetic field is given by:

$$
B_{g}=\frac{G \cdot\left[3 \cdot r \cdot(r \cdot S)-r^{2} \cdot S\right]}{c \cdot r^{5}}
$$

Eq. (1) shows that the ratio $\frac{\vec{v}}{c}$ is proportional to the non central force $\boldsymbol{F}_{L T}$. It therefore follows that the gravitomagnetic action of the rotation of the mass $M$ is proportional to the ratio $\frac{\vec{v}}{c}$. Acceleration $\gamma_{L T}$ caused by force $F_{L T}$ in the Newtonian evaluation can then be formulated as follows, where $\vec{\beta}$ is equal to $\frac{\vec{v}}{c}$ and $\frac{\vec{v}}{c}<<1$ :

$$
\overrightarrow{\gamma L T}=\frac{G \cdot M}{r^{2}} \cdot F(\vec{\beta})
$$

In the Newtonian context, this residual acceleration $\gamma \overrightarrow{L T}$ (orthogonal to the direction of angular momentum $\vec{S}$ and speed $\vec{v}$ ) caused by the Lense-Thirring effect, has a vector component along radius $r$ and the of $\beta$ function (projection of the vector $\vec{\beta}$ along radius $r$ ). Which combines with the Newtonian radial acceleration of a test particle of mass $m$ along radius $r$ to give total acceleration $\gamma$ as follows:

$$
\gamma=\frac{G \cdot M}{r^{2}} \cdot(1+f(\beta))
$$

If the frame of reference of test mass $m$ changes, it is as if $G$ (Newton's gravitational constant) takes the value $G_{\text {referential: }}$

$$
G_{\text {referential }}=G \cdot z(\beta)
$$

It therefore follows from Eq. (5) that $G$ will vary (Ivashchuk and Melnikov 2002; Melnikov 2007) with $z$ $(\beta)=1+f(\beta)$ as shown here: 


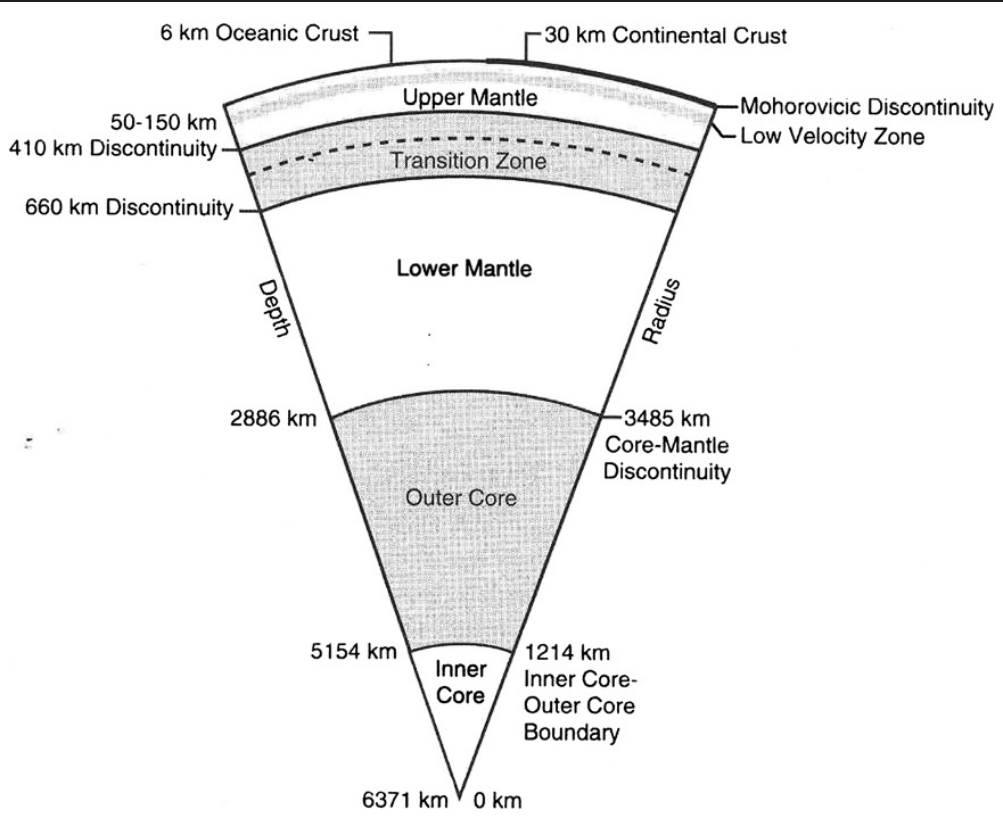

Figure 1 Simplified plan of the internal structure of the Earth.

$$
G_{\text {referential }}=G \cdot(1+k \beta) \text { with } f(\beta)=k \cdot \beta
$$

And the relativistic mass $M$ varies as a function of $\beta$ as shown here:

$$
M_{\text {relativistic }}=M \cdot\left(1-\beta^{2}\right)^{-\frac{1}{2}}
$$

Assuming that $k$ is equal to $1 / 2$ and the term $(1+k \beta)=1+\frac{1}{2} \cdot \beta \approx \sqrt{1+\beta}$ where $\beta$ is equal to $v / c$ and $v / c<<1$ (here $v$ is the projection of the vector $\vec{v}$ on the radial radius $\mathrm{r}$ ), replacing mass $M$ with its relativist value $M_{\text {relativistic }}$, total relative acceleration is given by:

$$
\gamma=\frac{G \cdot M}{r^{2}} \cdot \frac{1}{\sqrt{1-v / c}}
$$

Here $v$ is the projection of the vector speed $\vec{v}$ along the radial radius $r$.

It should be noted that if $G$ and $M$ are relativistic and depend on speed $v$, then $r$ does not depend on this speed and remains constant in this study.

An interesting characteristic of Eq. (8) is that acceleration is no longer independent of the sign of the velocity $v$ of the test particles making up the mass $M$ at the source of the gravitational field.

\section{Complete symmetry for the sun and the planets}

We now examine the case of gravitational masses, in particular the Solar System and the Sun whose volumetric expansion and mass are far greater than that of the planets. It is common knowledge that the planets revolve around a stationary Sun which itself rotates upon its axis. Over a short time span, the planets can also be considered as stationary in relation to the Sun and mass $\Delta M$ sun of the two hemispheres of the Sun moving with speed $+v$ or $-v$ in relation to the planets (Figure 2).

Speed $v$ is defined as the relative speed between the Sun's hemispheres and the planet in question, in this case the Earth. This does not take into account the influence of the other planets in the Solar System. The relative speed of the Sun's rotation seen by a test body $\Delta M x$ belonging to the Earth is almost zero because it is subject to speeds $+v$ and $-v$ of both hemispheres of the Sun (see Figure 2). Extended to the total mass of the Earth this speed is considered to have no effect on the action of the gravitomagnetic field of the Sun. By applying Eq. (8) and replacing $v$ by zero, acceleration $\gamma=\frac{G \cdot M}{r^{2}}$ is equal to Newton's classic relation.

However, this is not completely true as Figure 2 shows the distances $R 1$ and $R 2$ are not equal. Nevertheless, for the purposes of our application we will not take this into account. Applying the same reasoning used for the Sun to the Earth, Figure 3 shows the speeds of the Earth's hemispheres to be $+v$ and $-v$ (the speed of the Earth's rotation around its axis) and this relative speed is taken into account in the Eq. (8) of the acceleration $\gamma$ produced by the Sun on the element of mass $\Delta M x$ of the Earth.

Applying Eq. (8) and replacing $v$ by the speed, along the radius $r$, of the Earth's rotation, acceleration $\gamma$ takes the form:

$$
\gamma=\frac{G \cdot M}{r^{2}} \cdot \frac{1}{\sqrt{1-\frac{v}{c}}}
$$




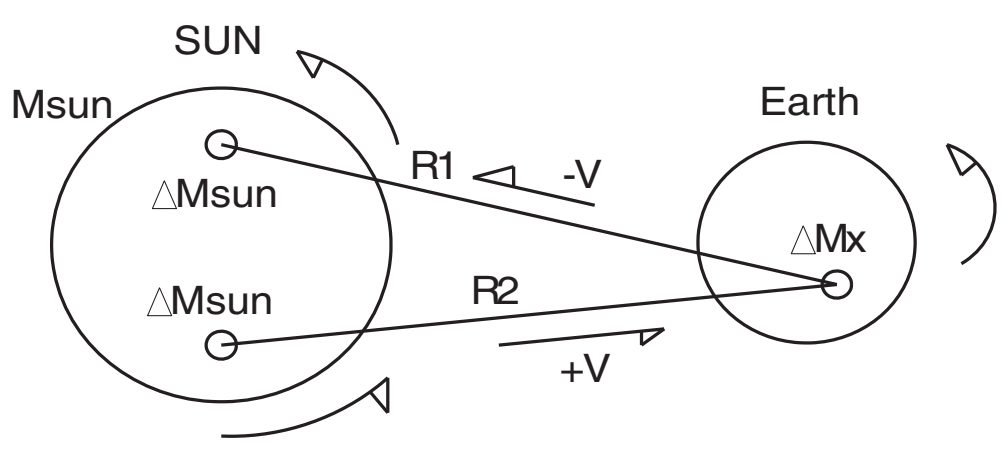

Figure 2 Cancellation of the Sun's rotation speed for mass $\Delta M x$.

\section{Definition of hidden variables}

Our calculations are based on a modified value for the radius of the Earth. This modification is described in Elbeze (2012). The Earth's real radius is defined as Rrr and the differential of the real radius as $d R r r$. As explained in Elbeze (op. cit.) the Earth, Sun, planets and the stars in general are complex systems and their apparent dimensions cannot be used directly in calculations.

This is a result of position-dependent hidden variables that maintain these celestial bodies in their respective planetary systems. In the case of the Earth the tilt of its rotation axis with respect to the ecliptic plane defines the real radius $R r r$ used here. Let us assume real radius $R r r$ for the Sun, and the apparent radius $R c b$ (cb for celestial bodies) for the planets of the Solar System, modified data $\Omega c b$ and experiential data $\Omega$ data, which is the projection of the sum of the angles of the axis of rotation and the angle of the orbit on the ecliptic plane.

$\Omega c b$ and $\Omega$ data consist of two data items: the orbital inclination $^{\mathrm{a}}$ (Seidelmann et al. 2007), which is the angle (in degrees) between the planet's orbit around the Sun and the ecliptic plane. The ecliptic plane is defined as the orbital plane of the Earth; therefore the Earth's inclination is 0. The second factor is the axial tilt ${ }^{\mathrm{a}}$ (Seidelmann et al. 2007), which is the angle (in degrees) between the rotational axis of a planet (the imaginary line running through the center of the planet from north to south poles) and its orbital axis around the Sun (see Figure 4).

$\Omega c b$ (Table 2) shows the corrected angle formed by the planet's rotational axis and the Earth's orbital plane (the ecliptic) used in our calculations. Obviously $\Omega c b$ must be unique for the celestial body in question. A global study of $\Omega$ data for orbital inclination and axial tilt led to the use of $\Omega c b$ as a variable.

The real radius $(R r r)$ can be written as:

$$
R r r=\frac{R c b}{1+62.3 \cdot \sin (\Omega c b)}
$$

And the differential $d R r r$ can be written as:

$$
d R r r \rightarrow \frac{d R c b}{1+62.3 \cdot \sin (\Omega c b)}
$$

$R c b$ corresponds to the apparent radius of the celestial body, here the Earth. From Eq. (9) and Eq. (10) it follows that the apparent radius $r$ can be replaced by:

$$
r \rightarrow \frac{r}{1+62.3 \cdot \sin (\Omega c b)} \quad \text { and } \quad d r \rightarrow \frac{d r}{1+62.3 \cdot \sin (\Omega c b)}
$$

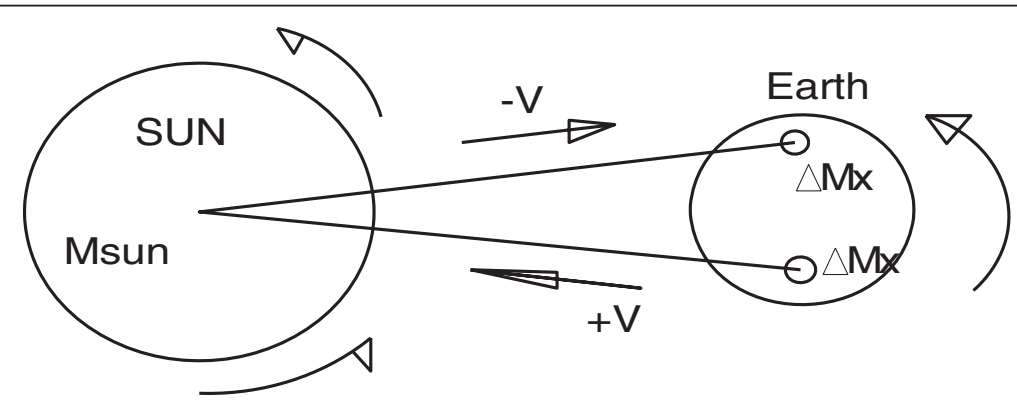

Figure 3 Relative speed of the Earth's rotation for mass $\Delta M x$. 


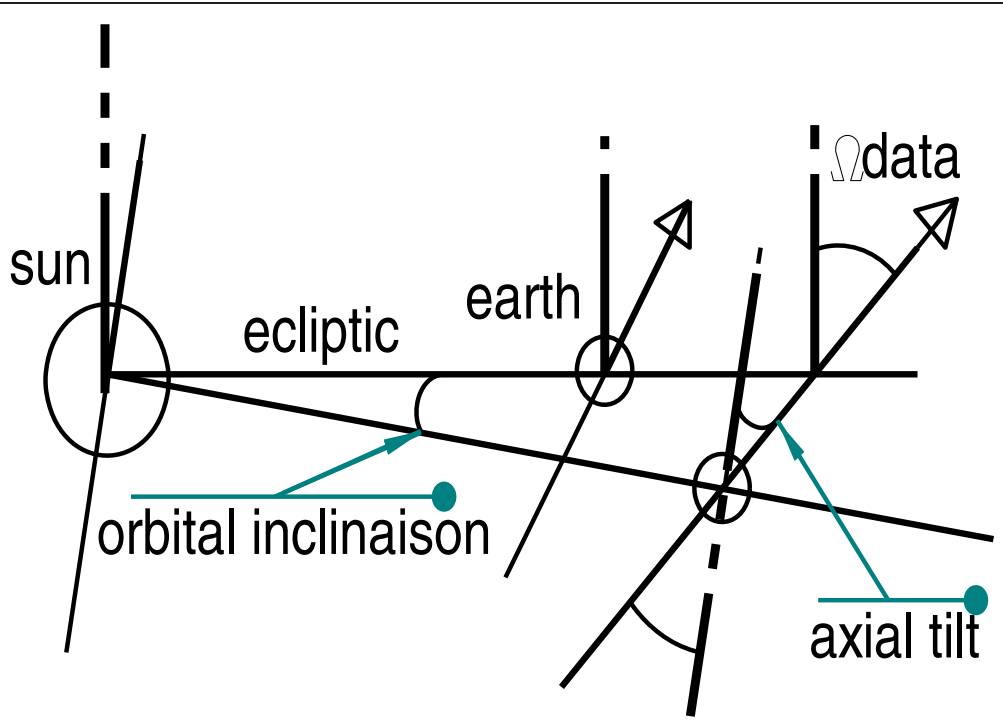

Figure 4 Definition of the angle $\Omega c b$.

This data makes it possible to calculate $\boldsymbol{H}_{\text {planet }}$ and $V_{\text {planet }}$ (see Figure 5) which are used later in the calculation, as follows:

$$
\text { Hplanet }=2 \cdot \frac{r}{1+62.3 \cdot \sin (\Omega c b)} \cdot \sin \theta \cdot \cos (\mathrm{O} c b)
$$

$\boldsymbol{H}_{\text {planet }}$ is the projection of the crown diameter on the axis which connects the center of the Sun to the center of the planet. $\boldsymbol{H}_{\text {planet }}$ is defined by the polar coordinates (Figure 6) $r$ and the angles $\theta, \phi$. O $c b$ represents the tilt of the axis of the Earth or of the planet on their orbital inclination. In the case of the Earth $O c b$ is equal to $\Omega c b$ (see Figure 5).

Table 2 Modifications of $\Omega$ data for the planets and the Sun (Elbeze 2012)

\begin{tabular}{lcc}
\hline i Planets & $\begin{array}{c}\text { Angle the ecliptic makes with } \\
\text { the projection of the axis of } \\
\text { rotation of the planet on the } \\
\text { ecliptic } \Omega \text { data (the data } \\
\text { observations in radians) }\end{array}$ & $\begin{array}{c}\text { modified } \Omega \text { data } \\
\text { as } \Omega \boldsymbol{c b} \text { in radians }\end{array}$ \\
\hline 0 Sun & 0.12654 & 0.00139 \\
1 Mercury & 0.12235 & $0.08221+\pi$ \\
2 Venus & 3.15556 & 3.12623 \\
3 Earth & 0.41015 & 0.45989 \\
4 Mars & 0.87092 & 0.81071 \\
5 Jupiter & 0.07679 & $0.45789+\pi$ \\
6 Saturn & 0.50964 & 0.71305 \\
7 Uranus & 1.72089 & 2.54469 \\
8 Neptune & 0.52534 & 0.5435 \\
\hline
\end{tabular}

In the following we calculate heat flow as a function of the latitude of the point in question. So far we have defined an effective radius $\mathrm{Rrr}$ which is function of $\Omega c b$ and the constant 62.3. This constant is only valid in the case of a planetary system where the projection of the angle $\Omega c b$ remains fixed on the ecliptic, as is the case for the Solar System. If we consider this variable angle $\Omega c b$ to the same planet along the ecliptic, as is the case in heat flow calculations that are a function of latitude, we need to define a function $f(\theta)$ to replace the constant. This fonction $f(\theta)$ is relatively easy to find and takes the following empirical form:

$$
f(\theta)=62.3 \cdot\left[0.52 \cdot \sin \left(\left(\frac{\pi}{2}-\theta\right) \cdot 2.45\right)+0.57\right]
$$

$\left(\frac{\pi}{2}-\theta\right)$ represents the latitude of the point considered.

It is clear that this function takes an approximate value of 62.3 for all the planets of the Solar System according to their angle $\Omega c b$ on the ecliptic (see Figure 7). Equations (11) and (12) can be reformulated as:

$$
\begin{gathered}
r \rightarrow \frac{r}{1+f(\theta) \cdot \sin (\Omega c b)} \text { and } d r \rightarrow \frac{d r}{1+f(\theta) \cdot \sin (\Omega c b)} \\
\text { Hplanet }=2 \cdot \frac{r}{1+f(\theta) \cdot \sin (\Omega c b)} \cdot \sin \theta \cdot \cos (\mathrm{O} c b)
\end{gathered}
$$




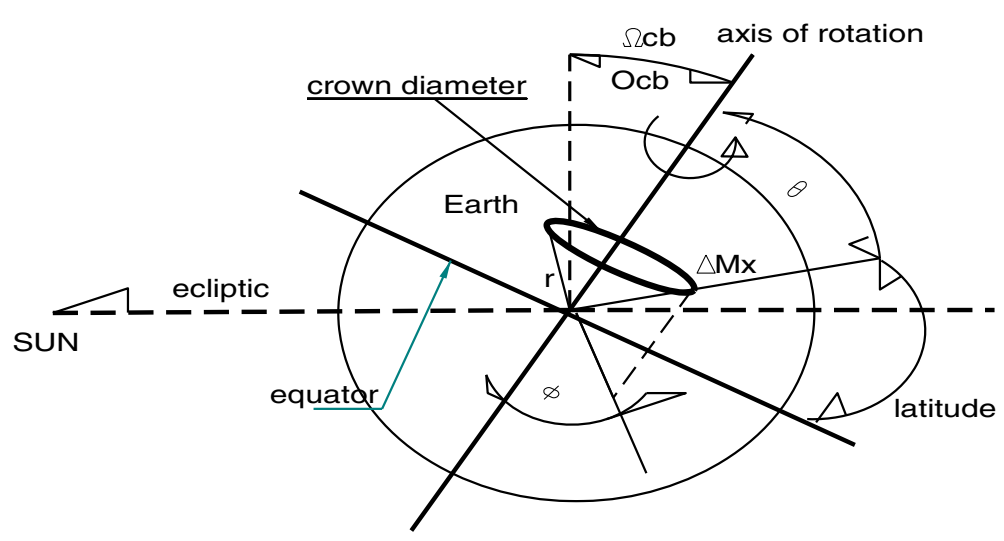

Figure 5 The Earth's tilt and the crown diameter.

In a short time frame (e.g. 24 hours) the speed of the Earth's rotation is constant, but its direction is variable. Consequently, it is easy to calculate the average relative velocity $V_{\text {planet }}$ when a mass $\Delta M x$ moves from the point farthest from the Sun to the nearest point. It can be represented as:

$$
\text { vplanet }=\frac{\text { Hplanet }}{12 \cdot 3600 \cdot \mathrm{sec}}
$$

$\boldsymbol{H}_{\text {planet }}$ is the distance traveled in 12 hours, half the time required for a complete revolution of the Earth, from the farthest to the point nearest to the Sun. It should be noted that the real radius $R r r$ is used to calculate $\boldsymbol{H}_{\text {planet }}$ and $\boldsymbol{V}_{\text {planet }}$. Both $\boldsymbol{H}_{\text {planet }}$ and $\boldsymbol{V}_{\text {planet }}$ are hidden variables, dependent on the position of the planet in the Solar System and its inclination to the ecliptic (Elbeze 2012).

\section{Reaction between the sun's gravitational field and the earth's rotation}

Using the polar coordinates and considering the Earth as having a quasi-continuous density in different parts from the inner core to the upper mantle we will consider mass $\Delta M x$ whose volume is defined according to the Figure 6.

Obviously, the radius used to calculate the volume and mass of $\Delta M x$ does not vary according to $\Omega c b$ as this is a real number and an apparent radius. $d V$ is calculated as follows:

$$
d V=r^{2} \cdot \sin \theta \cdot d r \cdot d \theta \cdot d \phi
$$

The infinitesimal mass $d m$ can be defined as:

$$
d M=\mu \cdot r^{2} \cdot \sin \theta \cdot d r \cdot d \theta \cdot d \phi
$$

Here $\mu$ is the density of the zone on the Earth and $r$ is the vector radius $r$ of the testing mass $\Delta M x$ (see Figure 6).

Next we calculate the effect of the gravitational field of the Sun on the mass $\Delta M x$ along its upward or downward trajectory of height $\boldsymbol{H}_{\text {planet. }}$ From Eq. (8) and using $d W=\int_{D 1}^{D 2} \gamma \cdot d M \cdot d r$ with $d W=d W u$ for upward and $d W=$ $d W d$ for downward for $D 1$ and $D 2$ this gives the equations below (16a and 16b).

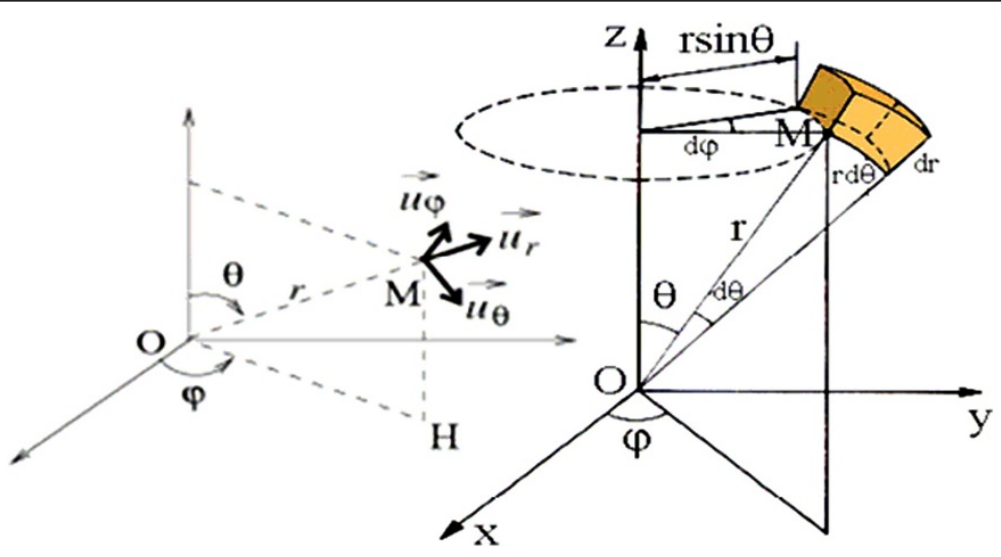

Figure 6 Polar coordinates of point $M$ of mass $\Delta M x$ on the Earth. 


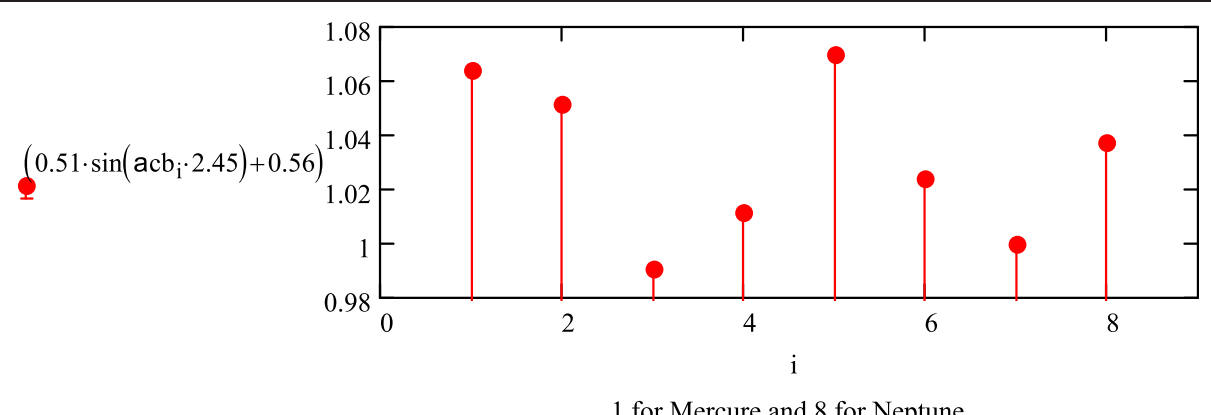

Figure 7 A calculation of $f(\theta)$ where $i=1$ for Mercury and $i=8$ for Neptune.

In order to facilitate the calculation, and not introduce large errors into the final results, the following considers $\boldsymbol{H}_{\text {planet }}$ and $\boldsymbol{V}_{\text {planet }}$ as acceptable average values. This is preferred to more accurate calculation that takes into account the infinitesimal displacement $\overrightarrow{d H p l a n e t}$ and variable speed $\overrightarrow{\text { Vplanet }}$.

Despite this simplification, which only incurs a slight quantitative error, we must bear in mind the fact that the speed $\overrightarrow{\text { Vplanet }}$.of the infinitesimal mass $\mathrm{dMx}$ has a direction which varies from 0 to $2 \pi$ over 24 hours.

Therefore, the gravitomagnetic force along the radius vector $\boldsymbol{H}_{\text {planet }}$ is not nul.

$$
\begin{aligned}
& D 1=\text { Dstar }-\frac{\text { Hplanet }}{2} \text { and } \\
& D 2=\text { Dstar }+\frac{\text { Hplanet }}{2} \text { for } d W u \\
& D 1=\text { Dstar }+\frac{\text { Hplanet }}{2} \text { and } \\
& D 2=\text { Dstar }-\frac{\text { Hplanet }}{2} \text { for } d W d
\end{aligned}
$$

Equations (16a) and (16b) define the two extreme points of the Earth from the center of the Sun

For $d W u$ with speed equal to $-v$ planet we have:

$$
d W u=\frac{G \cdot \text { Mstar } \cdot d M}{\sqrt{1-\frac{-v p l a n e t}{c}}} \cdot\left[\frac{-1}{\text { Dstar }+\frac{\text { Hplanet }}{2}}-\frac{-1}{\text { Dstar }-\frac{\text { Hplanet }}{2}}\right]
$$

For $d W d$ with speed equal to $v$ planet we have:

$$
d W d=\frac{G \cdot M \text { star } \cdot d M}{\sqrt{1-\frac{v p l a n e t}{c}}} \cdot\left[\frac{-1}{\text { Dstar }-\frac{\text { Hplanet }}{2}}-\frac{-1}{\text { Dstar }+\frac{\text { Hplanet }}{2}}\right]
$$

Where Dstar represents the distance from the center of the Sun to the center of the Earth, Mstar is the mass of the Sun, $G$ is Newton's constant and $c$ is the speed of light. The total gravitational energy of the reaction is given by:

$$
\begin{aligned}
W t & =\int_{0}^{2 \cdot \pi} \int_{0}^{\frac{\pi}{2}} \int_{0}^{\text {Rplanet }} \\
& \times(d W u+d W d) \cdot(24 \cdot 3600 \cdot \mathrm{sec})^{-1} \cdot \frac{1}{1+f(\theta) \cdot \sin (\Omega c b)}
\end{aligned}
$$

$W t$ (measured in watts or $J \mathrm{sec}^{-1}$ ) represents the total heat created by the gravitational action of the Sun on the planet, here the Earth. The term $(243600 \mathrm{sec})$ is the total time taken for a full rotation of the planet. $\frac{1}{1+f(\theta) \cdot \sin (\Omega c b)}$ represents the factor of $d r$ as shown earlier (Eq. 11). The calculation can be simplified as follows:

$$
\begin{aligned}
W t= & \int_{0}^{2 \cdot \pi} \int_{0}^{\frac{\pi}{2}} \int_{R 1}^{R 2} G \cdot \text { Mstar } \cdot(24 \cdot 3600 \cdot \mathrm{sec})^{-1} \\
& \times \frac{\text { Hplanet }}{\text { Dstar }^{2}-\left(\frac{\text { Hplanet }}{2}\right)^{2}} \cdot \\
& \times\left(\frac{1}{\sqrt{1-\frac{\text { vplanet }}{c}}}-\frac{1}{\sqrt{1-\frac{-v \text { planet }}{c}}}\right) \cdot \mu \cdot r^{2} \cdot \sin \theta \\
& \times \frac{1}{1+f(\theta) \cdot \sin (\Omega c b)} \cdot d r \cdot d \theta \cdot d \phi
\end{aligned}
$$

In reality, the planets of the Solar System travel along ecliptic orbits and Dstar should be replaced by Dstar = $a \cdot(1-e x \cdot \cos (\lambda))$ or $a=$ half major axis $=149,6 \cdot 10^{9} \cdot m$ For the Earth, $e x$ is the eccentricity $(0.0167)$ and $\lambda$ varies from 0 to $2 \pi$ ( 0 in winter and $\pi$ in summer; the farthest point of the Earth from the Sun).

The distance $R 1-R 2$ (from the center of the planet) represents the depth of the layer used to calculate the energy generated by the reaction with the gravitational field of the star. In the case of the Earth $R 1=0, R 2=$ Rearth and 
$\mu=5.515 \cdot 10^{3} \cdot \frac{\mathrm{kg}}{\mathrm{m}^{3}}$ which gives a value of $6.4 \cdot 10^{13} \cdot$ Watt for $W t$.

\section{Application of Wt to the earth}

We used data from Figure 1 and internal data densities to calculate the total heat production $W t$ at different depths as shown in Table 3 below:

Although the data used in Table 3 is relatively exact it clearly shows that the dimensions and densities are not completely accurate. If it was the case that the value of 32 TW (18 TW from the upper mantel and 14 TW from the lower mantel) had completely accounted for the $47 \mathrm{TW}$ heat contribution from the gravitational action of the Sun and the heat loss by the Earth, the heat contribution from radioactive elements would be in the order of $15 \mathrm{TW}$, which is comparable with the value generated by the Bulk Silicate Earth (BSE) model.

All of our calculations of Wt only take into account the action of the central star (the Sun) and the position of the Earth with respect to the Sun. However, the fact that the Earth takes an elliptic orbit around the Sun implies that $W t$ varies according to the Earth's position in space. At the same time, other planets in the Solar System have a gravitational effect on the Earth. This can be easily calculated by generalizing $W t$ and replacing the action of the Sun with that of other planets. The general application of $W t$ and with vplanet $\ll c$ can be written as:

$$
\begin{gathered}
W t=\int_{0}^{2 \cdot \pi} \int_{0}^{\frac{\pi}{2}} \int_{R 1}^{R 2} G \cdot M s t a r \cdot T c b^{-1} \cdot \frac{H c b}{D_{s t a r}{ }^{2}\left(\frac{H c b}{2}\right)^{2}} \\
\times \frac{v c b}{c} \cdot \frac{\mu c b \cdot r^{2} \cdot \sin \theta}{1+f(\theta) \cdot \sin (\Omega c b)} \cdot d r \cdot d \theta \cdot d \phi
\end{gathered}
$$

With:

$$
H c b=2 \cdot \frac{r}{1+f(\theta) \cdot \sin (\Omega c b)} \cdot \sin \theta \cdot \cos (\Omega c b)
$$

\begin{tabular}{|c|c|c|c|c|c|}
\hline \multicolumn{6}{|c|}{ Data on the Earth's Interior from Wt } \\
\hline & \multirow[t]{2}{*}{ Thickness (km) } & \multirow[t]{2}{*}{ Density $\left(\mathrm{g} / \mathrm{cm}^{3}\right)$} & \multicolumn{2}{|c|}{$\begin{array}{c}\text { Wt (10 } \\
\text { Watt) }\end{array}$} & \multirow[t]{2}{*}{$\pm \Delta$} \\
\hline & & & $\lambda=0$ & $\lambda=\pi$ & \\
\hline Crust & 30 & 2.2 to 2.9 & 0.84 & 0.69 & \pm 0.2 \\
\hline Upper mantle & 660 & 3.8 to 5.4 & 17.05 & 15.22 & \pm 1.5 \\
\hline Lower mantle & 2,226 & 5.2 to 6.2 & 32.40 & 30.40 & \pm 2.8 \\
\hline Outer core & 2,268 & 9.9 to 12.2 & 6.86 & 6.43 & \pm 0.5 \\
\hline Inner core & 1,217 & 12.8 to 13.1 & 0.04 & 0.036 & \\
\hline All the Earth & 6,371 & 5.5 & 59.50 & 56.00 & \pm 5 \\
\hline
\end{tabular}

Table 3 Depths, densities and heat production (Wt) of the Earth's interior

$\lambda$ defines the Earth's position relative to the Sun (see Eq. 27 below).

$$
v c b=H c b \cdot\left(\frac{T c b}{2}\right)^{-1}
$$

Where $c b$ is the celestial body subject to the gravitational field of mass Mstar (which generates the gravitational field), Dstar is the distance between the two bodies in question, $T c b$ is the round trip time of the celestial body, $H c b$ and $v c b$ concern the body subject to the gravitational field of the mass Mstar calculated using (Eq. 22) and (Eq. 23).

\section{Relationship between Wt and reduced heat flow}

In general we assume that the mantle and crust heat flux is proportional to the average surface heat flux. Pollack and Chapman (1977) argued that mantle heat flux represents $40 \%$ of the regional average surface heat flux. Despite the fact that their measures were based on a small dataset, we consider here that they are valid up to a minimum scale of about $300 \mathrm{~km}$ (Mareschal and Jaupart 2004). Average heat flow data suggest an empirical relationship of the form:

$$
\bar{Q}=Q o+b \cdot \bar{H}
$$

where $\bar{Q}$ and $\bar{H}$ represent average heat flux across the designated area and heat production, $Q o$ is reduced heat flow and $b$ represents the thickness of a shallow layer enriched by radiogenic elements. Eq. (24) reflects

\begin{tabular}{|c|c|c|c|c|}
\hline $\mathrm{N}^{\circ}$ & Terrain & $\begin{array}{l}\text { Reduced Heat } \\
\text { Flow } \mathrm{mW} / \mathrm{m}^{2}\end{array}$ & Latitude & References \\
\hline 1 & Baltic Shield & 24 & $66 \mathrm{~N}$ & Balling, 1995 \\
\hline 2 & Brazil Coastal & 43.152 & $25 \mathrm{~S}$ & $\begin{array}{l}\text { Vitorello } \\
\text { et al., } 1980\end{array}$ \\
\hline 3 & $\begin{array}{l}\text { Central } \\
\text { Australia }\end{array}$ & 48.8 & $23 \mathrm{~S}$ & $\begin{array}{l}\text { McLaren } \\
\text { et al., } 2001\end{array}$ \\
\hline 4 & $\begin{array}{l}\text { Eastern USA } \\
\text { Phanerozoic }\end{array}$ & 26.961 & $40 \mathrm{~N}$ & $\begin{array}{l}\text { Roy et al., } \\
1968\end{array}$ \\
\hline 5 & $\begin{array}{l}\text { Eastern USA } \\
\text { Proterozoic }\end{array}$ & 26.524 & $41 \mathrm{~N}$ & $\begin{array}{l}\text { Roy et al., } \\
1968\end{array}$ \\
\hline 6 & Fennoscandia & 23.5 & $54 \mathrm{~N}$ & $\begin{array}{l}\text { Kukkonen } \\
\text { et al., } 2001\end{array}$ \\
\hline 7 & Maritime & 34.54 & $30 \mathrm{~N}$ & $\begin{array}{l}\text { Hydman } \\
\text { et al., } 1979\end{array}$ \\
\hline 8 & Piedmont & 28.61 & $37 \mathrm{~N}$ & $\begin{array}{l}\text { Costain et al., } \\
1986\end{array}$ \\
\hline 9 & Ukraine & 24.32 & $49 \mathrm{~N}$ & kutas, 1984 \\
\hline 10 & Wyoming & 26.25 & $42 \mathrm{~N}$ & $\begin{array}{l}\text { Decker et al., } \\
1988\end{array}$ \\
\hline 11 & Yilgarn & 33.334 & $32 \mathrm{~s}$ & Jaeger, 1970 \\
\hline
\end{tabular}
changes in average heat flux on a larger scale $(>200 \mathrm{~km})$ and is based on a very large dataset. It implies that

Table 4 Reduced heat flows for the linear data fit of individual terrain 


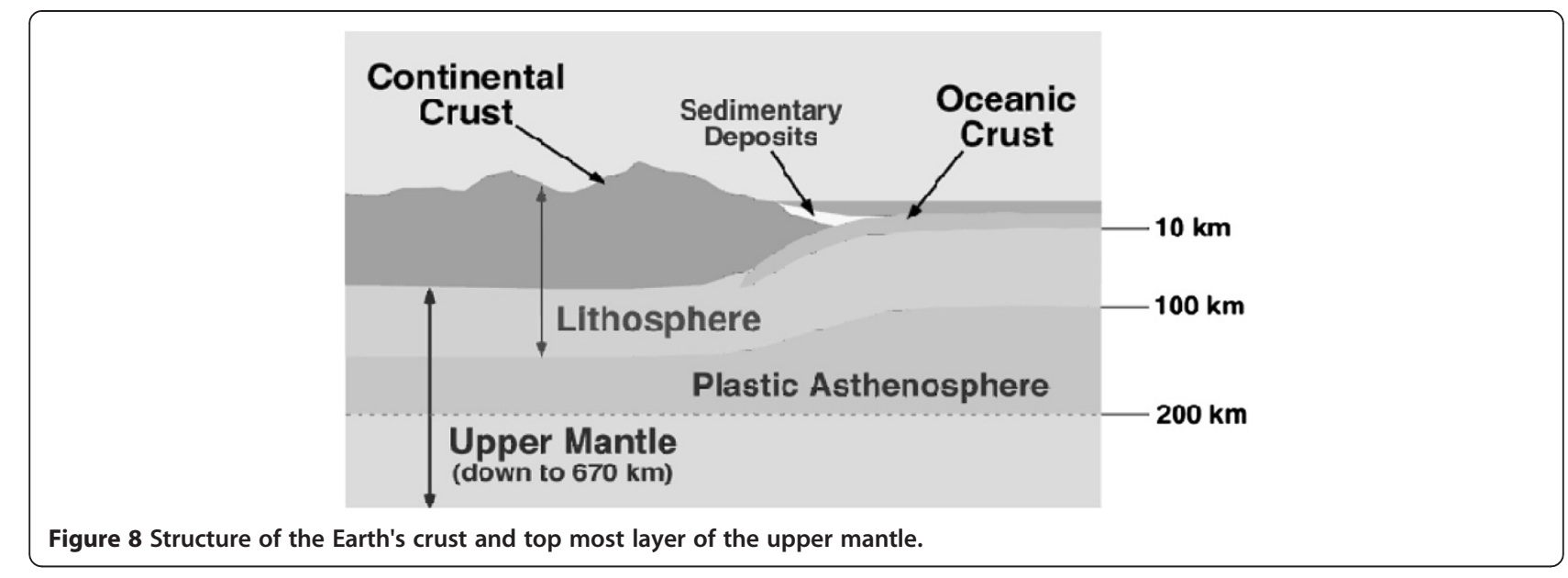

reduced heat flux Qo is the same at a certain depth and latitude ${ }^{\mathrm{b}}$ of the crust in all areas. The assumed value $Q o$ is clearly shown in Eq. (21), which expresses the gravitational action of the Sun on the Earth. This data can be checked against the data provided in Table 4 (above), from the study by Thakur and Blackwell, Huffington Department of Earth Sciences, Southern Methodist University, Dallas, TX.

In order to compare Eq. (21) with the value of Qo (reduced heat flow), we must extend Eq. (21) which calculates heat production due to the gravitational action of the Sun on the Earth, to calculate heat flow up to depths of the order of $500 \mathrm{~km}$, comprising the lithosphere and the upper mantel.

Taking $\theta$ and $\phi$ to represent the latitude and longitude of the area where average heat flow is measured, this relation can be written as follows, with the latitude measured in degrees:

$$
\theta=\pi \cdot \frac{90-\text { latitude }}{180} \text { and } \phi=\frac{\pi}{180} \cdot \text { longitude }
$$

The calculation of the gravitational action of the Sun on the Earth is shown in Eq. (21). However, for the calculation of heat flow and heat production, we will base the calculation on a $1 \mathrm{~m}^{2}$ column in a lithosphere approximately $550 \mathrm{~km}$ deep. In this area, heat transfer mainly occurs through thermal conduction, which enables us to assume that the heat produced as a result of the gravitational effect of the Sun in this area is equal across wide areas and therefore comparable to the reduced heat flow shown in Eq. (24). Heat propagation is lowest in the lower mantle; it is no longer completely the result of thermal conduction but various according to the geography of the area and is comparable to heat production $\bar{H}$ shown in Eq. (24). We will see later that only a portion of the heat

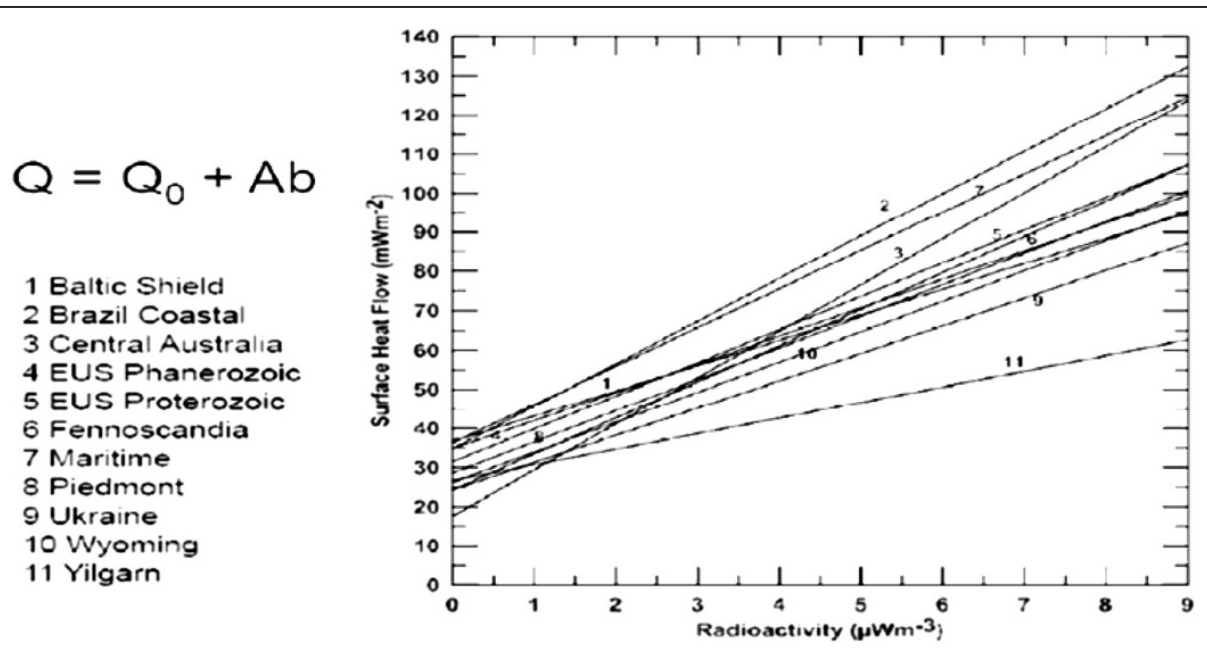

Figure 9 The linear fit line for individual Q-A data for different terrain of the world. 


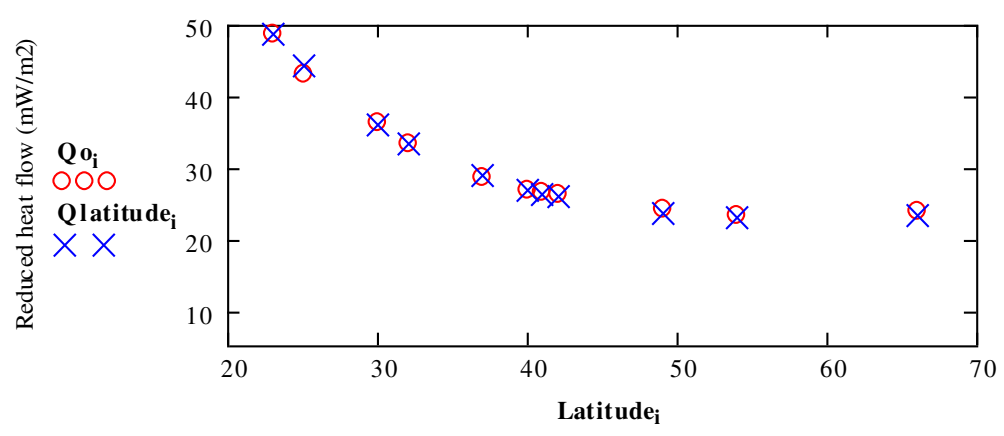

Figure 10 Application of $Q$ (latitude) in regards to the data Qoi.

production generated by the gravitational field of the Sun in the lower mantel is taken into account in the calculation of the Earth's heat loss.

Other explanations for the Earth's internal heat can be found on the Internet.

We can write:

$$
\begin{aligned}
W t= & \int_{\phi-\Delta \phi}^{\phi} \int_{\theta-\Delta \phi}^{\theta} \int_{R 1}^{R 2} G \cdot M s t a r \cdot T c b^{-1} \\
& \times \frac{H c b}{(\text { Dstar } \cdot(1-E X c b \cdot \cos (\lambda)))^{2}-\left(\frac{H c b}{2}\right)^{2}} . \\
& \times \frac{v c b}{c} \cdot \frac{\mu c b \cdot r^{2} \cdot \sin (\theta)}{1+f(\theta) \cdot \sin (\Omega c b)} \cdot d r \cdot d \theta \cdot d \phi
\end{aligned}
$$

Where EXcb is the eccentricity of the Earth's orbit or the planet's orbit (cb indicating a celestal body), and the angle $\lambda$ varies from January to August from 0 to $\pi$ with Dstar $=1.496 \cdot 10^{11} m=a$, where $a$ is equal to half major axis of the eliptic orbit of the planet, and:

$$
\begin{aligned}
& \Delta \phi=\arctan \left(\frac{\Delta R}{r \cdot \sin (\phi)}\right) \text { and } \\
& \Delta \theta=\arctan \left(\frac{\Delta R}{r}\right)
\end{aligned}
$$

$\Delta R$ is a basic unit of distance. It can be a meter or take an arbitrary value. Here, we use the meter because it is directly related to the unit area $m^{2}$ or the unit volume $m^{3}$ which leads to a definition of heat flow and heat production in $\left(m W / m^{2}\right.$ or $\mu W / m^{3}$.).

Finally, heat production $\bar{H}$ of the gravitational action of the Sun on the Earth can be written as follows:

$$
\bar{H}=W t(R 1, R 2, \mu, \lambda, \phi, \theta, \Delta \phi, \Delta \theta)
$$

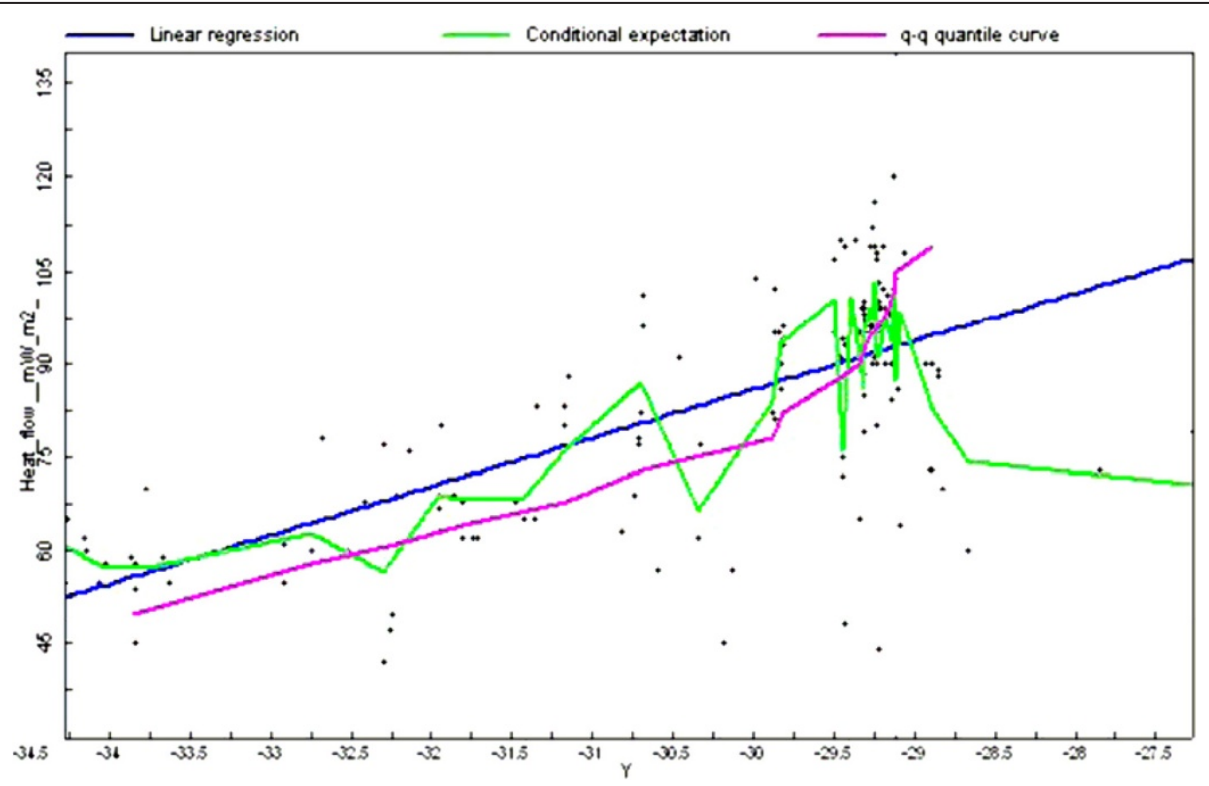

Figure 11 A heat flow values ( $y$ axis) and latitude ( $x$ axis). 


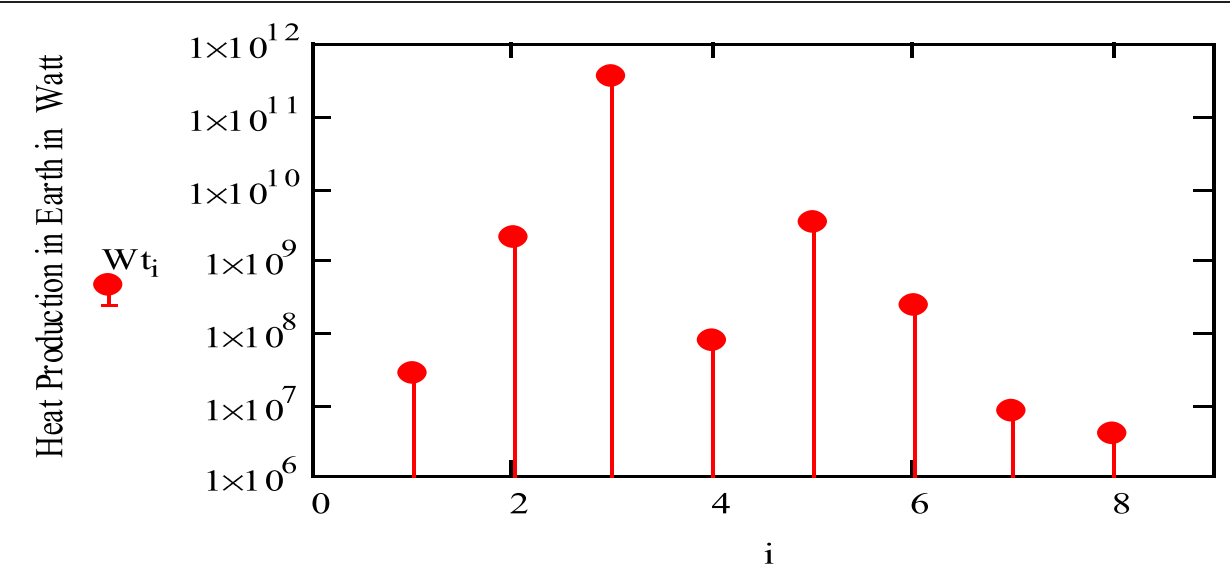

Figure 12 Calculation of the gravitational action of planets on the Earth $(W t)$.

For example, according to Eq. (28) at $23^{\circ}$ north, heat production from the gravitational action of the Sun on the Earth can be written as:

$$
\begin{aligned}
& R 1=R_{\text {Earth }} \quad \lambda=0 \text { and } \phi \text { any } \\
& R 2=R_{\text {Earth }} \\
& \mu=\frac{\text { Mearth }}{1.6}=3.447 \mathrm{Kg} \cdot \mathrm{m}^{-3} \\
& \bar{H}\left(\text { latitude } 23^{\circ}\right)=\operatorname{Wt}(R 1-\Delta R, R 2, \mu, \lambda, \phi, \theta, \Delta \phi, \Delta \theta) \\
& \times \frac{1}{\Delta R^{3}}=0.095 \cdot \mu \mathrm{W} \cdot \mathrm{m}^{-3}
\end{aligned}
$$

In Eq. (29), $\frac{1}{\Delta R^{3}}$ enables heat production and heat flow in a volume of $1 \mathrm{~m}^{3}$ to be calculated. Consequently, for a lithosphere of thickness $558 \mathrm{~km}$ (Pinet C et al. (1991)) at $23^{\circ}$ north the calculation is as follows:

$$
\begin{aligned}
& Q\left(\text { latitude } 23^{\circ}\right) \\
& =\int_{\text {Rearth-thickness }}^{\text {Rearth }} W t(R 1-\Delta R, R 2, \mu, \lambda, \phi, \theta, \Delta \phi, \Delta \theta) \\
& \times \frac{1}{\Delta R^{3}} \cdot d r=48.734 \cdot m W \cdot m^{-2}
\end{aligned}
$$

This value of $558 \mathrm{~km}$ is close to the $660 \mathrm{~km}$ depth of the Earth's lithosphere and upper mantel (see Figure 8) and $\lambda=0$ (see Eq. 26).

If $\boldsymbol{Q}$ (latitude) is applied to the data in Table 4, and Figure 9, we can check whether $Q$ (latitude) does in fact represent the reduced heat flow Qo found in Eq. (24) for regions at different latitudes. In fact Figure 10 shows that the gravitational action of the surrounding planets and the Sun heat the Earth in the same proportions as the reduced heat flow Qo.

Earlier literature on the Earth's heat sources has also suggested the possibility of an external heat source. For example, Jaupart et al. (2007) comment that Qo could be due to an external input of heat and differences of the radiogenic heat of the Earth's crust.

It is interesting to note that Figure 10 shows that reduced heat flow decreases at higher latitudes. This is not unusual; several other authors have noted this phenomenon. For example, Figure 11 shows results from a study of the north-northwest of Western Australia (Perth Basin) carried out by the company Hot Dry Rocks Pty Ltd (2008).

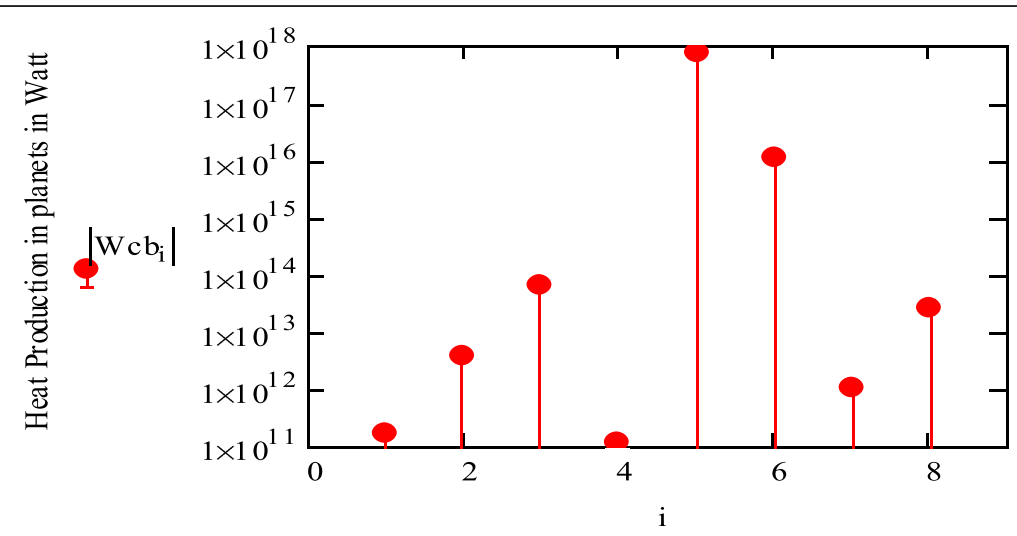

Figure 13 Calculation of $W t$ the gravitational action of the Sun on the planets. 


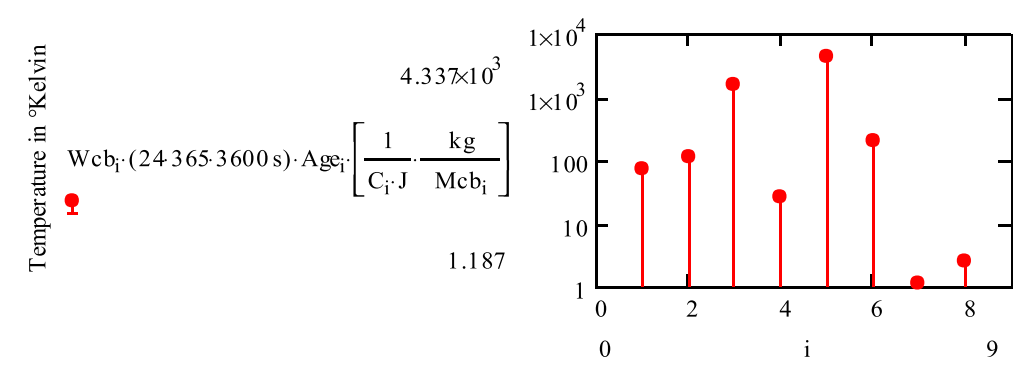

Figure 14 Calculation of the gravitational action of the Sun on the planets.

\section{Reaction between the planets' gravitational field and the earth's rotation}

From Eq. (21), we can calculate the gravitational effect of the planets and the Moon on the Earth. To do this we replace the data relative to the effect of the Sun on the Earth with those of the planet or satellite in question (e.g. the Moon). According to Table 2 the values to be used are $i=1$ for Mercury, $i=8$ for Neptune and $i=3$ for the Moon.

The graph shown in Figure 12 takes into account the minimum distance between the planet and the Earth (i.e. the distance from the Sun to the planet -the distance from the Earth to the Sun).

These calculations assume that the distances between the planets and the Earth remain constant over the period of the Earth's rotation. Although we know that this is not the case, the values of $W t$ shown in Figure 12 provide a relatively precise glimpse of the heat generated in the Earth. The effect of the Moon is the strongest, generating about $1 \mathrm{TW}$ (particularly compared to tidal dissipation in solid earth of about $0.1 \mathrm{TW}$ ).

As before, we can calculate the gravitational action of the Sun on the planets in the Solar System. To do this, we apply Eq. (21), taking into account eccentric planetary orbits, to the relationship described in Eq. (26). The results are shown in the graph (Figure 13).

We can then calculate the temperature brought about by the gravitational action of the Sun on the planets of the Solar System for planets aged about $5 \cdot 10^{9}$ years. Using Eq. (21) we obtain the graph (Figure 14).

$C_{i}$ is heat capacity measured in $J \mathrm{~kg}^{-1} \cdot \mathrm{K}^{-1} ; \mathrm{Mcb}$ is the mass of the planet or celestial body. The data shown in this graph is imprecise as the value of $C_{i}$ is not well established.

\section{Conclusion}

Extending earlier studies on the rotation of planets (Elbeze 2012) and particularly the relativistic effect of gravitational action (see Eq. 8 and following) this paper shows that there is another heat source, external to the Earth itself and the action of its radioactive elements. Our calculations suggest that the gravitational effect of the Sun on the Earth generates a total power equal to about 54 TW.
This external heat is due to the action of land masses moving in the gravitational field of the Sun, and depends on the relative speed $\pm v$ (velocity depends on the rotation of the Earth on its axis). This occurs because there is an asymmetry between the direction of the relative speed and its effect on the moving masses (as described by Eq. 8 and following). Infact it is an example of the gravitomagnetism phenomenon described in the study by Elbeze (2012).

This study has shown that the production of heat in the lithosphere exactly matched the reduced heat flow Qo shown in Figure 10, and the heat lost from the lower mantle forms part of the overall heat lost by the Earth. However, if the heat flow created in the lower mantel does form part of the total $47 \mathrm{TW}$ of heat lost by the Earth, then heat produced by radioactive substances in the upper mantle must be less than the current estimate of 20 TW. Similarly, if the gravitational action of the Sun in the lower mantel creates a heat loss by the Earth of about $14 \mathrm{TW}$, the production of radiogenic heat would be about $15 \mathrm{TW}$ (or less), which is comparable with estimates based on the Bulk Silicate Earth value (BSE) model. The 14 TW produced by the gravitational action of the Sun on the Earth would vary from one area to another depending on the distribution of sedimentary rocks. This energy would add to the part of the heat produced by radioactivity found in the crust and the lower and upper mantels to form the heat flow lost by the Earth. In this case, the balance of the Earth's heat production would be positive, rather than negative. The overall effect of the gravitational action of the Sun on the Earth would be to increase heat generation by about 25 TW (see Table 3), which corresponds to an increase in temperature of the order of $170^{\circ} \mathrm{K}$ per billion years.

\section{Endnotes}

${ }^{\text {a }}$ Planetary fact sheet; can be found on the Internet.

${ }^{b}$ Latitude is a geographic coordinate that specifies the north-south position of a point on the Earth's surface. Lines of constant latitude (parallels) run east-west parallel to the Equator. Latitude is an angle which ranges from $0^{\circ}$ at the Equator to $90^{\circ}$ at the north and south poles. 


\section{Competing interests}

The author declared that he has no competing interests.

Received: 7 May 2013 Accepted: 25 September 2013 Published: 5 October 2013

\section{References}

Einstein A (1950) The meaning of relativity. Princeton University Press, Princeton, NJ

Elbeze AC (2012) On the origin of the spin of planets and stars and its connection with gravitomagnetism. Springer: Earth Moon Planets, DOI 10.1007/s11038-012- 9388-9

Bellini G et al (2010) Observation of geo-neutrinos. Phys Lett B 687:299-304

Davies JH, Davies DR (2010) Earth's surface heat ux. Solid Earth 1:5-24, www.solid-earth.net/1/5/2010/

De Sitter W (1916a) On Einstein's theory of gravitation, and its astronomical consequences. Mon Not R Astron Soc 76:699-728

Jaupart C, Labrosse S, Mareschal J-C (2007) Temperatures, heat and energy in the mantle of the earth. In: Bercovici D (ed) Treatise on geophysics, Vol. 7, mantle convection. Elsevier, pp 253-303

Mareschal JC, Jaupart C (2004) Variations of surface heat ow and lithospheric thermal structure beneath the north American craton. Earth Planet Sci Lett 223:65-77

Mareschal JC, Jaupart C, Cheng LZ, Rolandone F, Gari_epy C, Bienfait G, Guillou-Frottier L, Lapointe R (1999) Heat ow in the trans-Hudson orogen of the Canadian shield: implications for proterozoic continental growth. J Geophys Res 104:29,007-29,024

McDonough WF, Sun S-s (1995) The composition of the earth. Chem Geol 120:223-253

Ruggiero ML, Tartaglia A (2002) Gravitomagnetic effects. II Nuovo Cimento B 117(07):743

Palme H, O'Neil HSC (2003) Cosmochemical estimates of mantle composition. In: Carlson RW (ed) Treatise on geochemistry, vol. 2, mantle and core. Elsevier

Pinet C, Jaupart C, Mareschal JC, Gari_epy C, Bienfait G, Lapointe R (1991) Heat ow and structure of the lithosphere in the eastern Canadian shield. J Geophys Res 96:19,941-19,963

Pollack HN, Hurter SJ, Johnson JR (1993) Heat-flow from the earth's interior analysis of the global data set. Rev Geophys 31:267-280

Pollack HN, Chapman DS (1977) On the regional variation of heat flow, geotherms, and lithospheric thickness. Tectonophys 38:279-296

Seidelmann PK, Archinal BA, A'Hearn MF, Conrad A, Consolmagno GJ, Hestroffer D, Hilton JL, Krasinsky GA, Neumann G, Oberst J, Stooke P, Tedesco EF, Tholen DJ, Thomas PC, Williams IP (2007) Celest Mech Dyn Astron 98:155

Ivashchuk VD, Melnikov VN (2002) Problems of G and multidimensional models. In: Koga J et al (eds) 405-409 Proceedings of JGRG11. Waseda University, Tokyo, pp 405-409

Melnikov VN (2007) Variations of constants as a test of gravity, cosmology and unified models. Grav Cosmol 13(50):81-100

doi:10.1186/2193-1801-2-513

Cite this article as: Elbeze: On the existence of another source of heat production for the earth and planets, and its connection with gravitomagnetism. SpringerPlus 2013 2:513.

\section{Submit your manuscript to a SpringerOpen ${ }^{\circ}$ journal and benefit from:}

- Convenient online submission

- Rigorous peer review

- Immediate publication on acceptance

- Open access: articles freely available online

- High visibility within the field

Retaining the copyright to your article

Submit your next manuscript at $>$ springeropen.com 